\title{
Tumor dedifferentiation: diagnostic and therapeutic implications
}

\author{
Jha Abhimanyu ${ }^{1}$

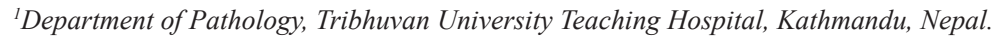

\section{Keywords: \\ Carcinoma; \\ Dedifferentiation; \\ Liposarcoma; \\ Melanoma; \\ Sarcoma;}

\begin{abstract}
Some of the neoplasm especially malignant tumors are notorious in masquerading their cell of origin because of additional mutations which drives them to differentiate into unusual phenotype. This is implicated to a phenomenon of tumor dedifferentiation which can mislead into inappropriate categorization and therapy. Dedifferentiation is well recognized in sarcomas such as liposarcoma, chondrosarcoma and malignant peripheral nerve sheath tumor. However, it can also develop in carcinomas, melanomas and lymphomas at initial diagnosis, following therapy or at recurrence. The phenomenon has been reported in both primary tumors as well as at metastatic foci. A correct and early pathological identification of this phenomenon might profoundly help in guiding appropriate therapy. Clinical and radiological findings, immunohistochemistry and genetic analysis are often required for correct lineage identification of these tumors.
\end{abstract}

\section{Correspondence:}

Dr. Abhimanyu Jha, MD

Associate Professor, Department of Pathology

Tribhuvan University Teaching Hospital, Maharajgunj, Kathmandu, Nepal ORCID ID: 0000-0003-4477-6129

Email: jhaabhimanyu@yahoo.com

Reveived : July 15th, 2017 ; Accepted : August 8th, 2017; Published : September 1, 2017

Citation: Jha A. Tumor dedifferentiation: diagnostic and therapeutic implications. J Pathol Nep. 2017;7: 1202-11. doi: 10.3126/jpn.v7i2.18021

Copyright: This is an open-access article distributed under the terms of the Creative Commons Attribution 4.0 International License, which permits unrestricted use, distribution, and reproduction in any medium, provided the original author and source are credited.

\section{INTRODUCTION}

Malignant tumors are notorious in masquerading their cell of origin, because of additional mutations, which drives them to differentiate into unusual phenotype. This is implicated to a 'phenomenon' of tumor 'dedifferentiation' which can mislead into inappropriate categorization and therapy. Dedifferentiation is well recognized in sarcomas. However, it can develop in carcinomas, lymphomas and melanomas. ${ }^{1-3}$ Dedifferentiation can arise de novo (combined with well differentiated tumor) at initial diagnosis, or develop at recurrence or at metastatic site and may follow therapy. A correct and early pathological identification of this phenomenon might profoundly help in guiding appropriate therapy. Clinical, radiological, immunohistochemistry and genetic analysis are often required for correct lineage identification of these tumors. This review will briefly highlight the mechanisms of tumor dedifferentiation in different types of malignant tumors, the 
diagnostic dilemmas, and therapeutic implications.

\section{DIFFERENTIATION AND DEDIFFERENTIATION}

Differentiation is a biological process by which a primitive cell develop or evolutes into a mature cell within same lineage to serve a specialized function. The conventional concept was that differentiation is unidirectional and once a cell differentiates to mature cell type, it never reverts back, like ball rolling down a hill into a landscape. ${ }^{4}$ A primitive cell once becomes mature it never reverts back to stem cell again. ${ }^{4}$ This concept may not be applicable now as by artificial reprogramming process a mature cell can be sent back to stem cell state. ${ }^{4}$ In tissue regeneration a terminally differentiated cell reverts back to a less differentiated stage from within its own lineage to proliferate and re-differentiate again to replace the lost cells. ${ }^{5}$

Dedifferentiation is a reprogramming process with morphological loss of lineage identity. ${ }^{6}$ The term is applicable to a situation where a more specialized cell type loses expression of lineage specific genes of specialized tissue function and acquires expression of genes which is seen in primitive state of the related tissue development., This genetic alteration in tumor cells results in acquisition of stem cell phenotype. ${ }^{7,8}$

\section{DIFFERENTIATION A BIDIRECTIONAL PROCESS}

Biologists have successfully turned back the clock on cells, to convert the mature cells into pluripotent stem cells, which can then differentiates into any cell lineage. This has been one of the most significant developments in field of stem cell research. ${ }^{9}$ Nobel prize for 2012 in Medicine was awarded to Sir John B. Gurdon and Dr. Shinya Yamanka for the discovery that mature cells can be reprogrammed to become pluripotent cells. ${ }^{9}$

The current experiment on induced stem cells (iPSC) has proved that differentiation of cells is bidirectional (fig.1). ${ }^{4}$ It is proven that a somatic nucleus can be reprogrammed to embryonic like state (pluripotency) by introducing four genes called Yamanaka factors (Oct 3/4, Sox2, Klf4, c-Myc). ${ }^{9-11}$ They are highly expressed in embryonic stem cells. Forced induction of these genes results in generation of iPSC. ${ }^{10,11}$ A pluripotent cell can differentiate to any lineage and give rise to different cell types. For example a pluripotent cell can differentiate to fibroblast, neuron, exocrine cell of pancreas, beta cell of pancreas and cardiac myocytes. ${ }^{5}$ A reprogramming process can induce a differentiated cell into pluripotent state which in other hand can differentiate into any of cell types. For example mature fibroblasts can be converted into pluripotent cells by the help of genes OCT4, SOX2, KLF4 and MYC. ${ }^{5}$ Neural stem cell can be reprogrammed to pluripotent stem cells by using gene OCT4. Cells can also change the lineage to another cell type and this phenomenon is called transdifferentiation.
A pancreatic exocrine cell when reprogrammed with genes PDX1, NGN3 and MAFA can transdifferentiate into beta cell. $^{5}$

Almost any differentiated cell can be reprogrammed to pluripotent state by using appropriate transcription factors and many of them are oncogenes. This concept is also applicable in phenomenon of origin of cancer stem cells. There are similarities between somatic reprogramming and tumor dedifferentiation. ${ }^{7}$

\section{CANCER STEM CELL AND INDUCED STEM CELLS}

The process of dedifferentiation is involved in both initiation and promotion of carcinogenesis. ${ }^{4}$ The phenomenon of reprogramming of somatic cells into iPSC is also a process of dedifferentiation, though it is induced experimentally. ${ }^{4}$ iPSCs and cancer cells not only share the properties of limitless replicative potential and self-renewal, but also shares similar metabolic status, that are acquired by somatic cells during reprogramming., ${ }^{42,13}$ Carcinogenesis and reprogramming of somatic cells, both require similar genetic alterations. This is also evident by the fact that iPSCs generation is significantly promoted by genetic ablation of TP53 which is major tumor suppressor gene to be mutated in carcinogenesis. ${ }^{4,14,15}$

The continuous growth and maintenance of many normal tissues such as skin, gastrointestinal epithelium and hematopoietic cells in bone marrow depends on resident population of stem cells that are capable of self renewal. With this background it easy to understand that cancer cells are immortal and have limitless proliferative capacity, they must contain cells that self-renew, the cancer stem cells. ${ }^{16}$ Cancer stem cells (CSCs) are biologically unique cells within a tumor, possessing self renewal capacity and produce progeny with self proliferative capacity, similar to normal stem cells. ${ }^{7}$ Cancer stem cells can perpetuate indefinitely unlike bulk of cells that are present in the tumor. ${ }^{7}$ Cancer stem cells is a cell that divides to replenish a population of cancer cells. ${ }^{17}$

The concept of CSCs has important implications for cancer therapy. If CSCs are essential for persistence of cancer, these cells must be eliminated to cure the cancer. Cancer stem cells have high intrinsic resistance to conventional therapies due to low proliferative capacity and expression of multi drug resistance-1 (MDR1) that counteract the effects of chemotherapy. This is the reason for limited success in current cancer treatment due inability to eradicate Cancer stem cells. ${ }^{7,16}$

Cancer stem cells can arise from transformation of tissue stem cells or from conversion of conventional somatic cell to transformed cells which acquire property of stem cells. Over that cancer cells within a tumor may be able to 


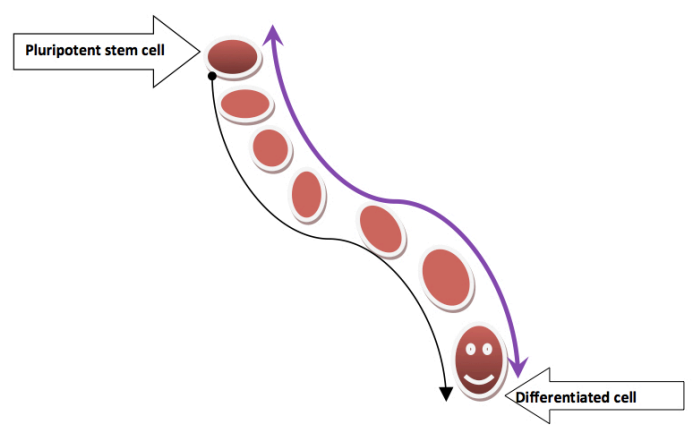

Figure 1: Pluripotent stem cell at the top (hill) and differentiated cells at bottom (valley). The traditional concept of unidirectional differentiation (curved arrow on left). Current concept of bidirectional differentiation (curved arrow on right).

dedifferentiate to a stem cell like state. ${ }^{16}$ Thus, although, CSCs poses properties of self renewal and differentiation similar to normal stem cells, they do not necessarily originate from the transformation of normal tissue stem cells. ${ }^{7}$ CSCs are often the cells that leave the primary tumor, survive in the blood stream, and metastasize to distant organs. ${ }^{17}$

\section{CANCER STEM CELLS AND TUMOR DEDIFFERENTIATION}

Normal stem cells can get mutated to get transformed into CSC, however, by this process high rate of mutation is required. ${ }^{18}$ The differentiated cells within the tumor can incur mutation to dedifferentiate into cancer stem cells. ${ }^{18}$

When a stem cell divides to produce a stem cell and a progenitor cell, this is called asymmetric division. If a stem cell divides to produce two stem cells, then this is called symmetric division. ${ }^{17}$ A progenitor cell is similar to a stem cell, except that it has less potential with regard to what cell types it can mature to become. ${ }^{17}$ Effect of dedifferentiation on carcinogenesis depends on how body controls the stem cells (homeostasis). ${ }^{18}$ When all the divisions of stem cells are asymmetric that is a tight homeostasis, dedifferentiation has little effect on carcinogenesis, and however, if the homeostasis is loose, that is both symmetric and asymmetric division, dedifferentiation has rapid effect on carcinogenesis and lead to exponential growth of cancer stem cell population. ${ }^{18}$

Bulk of tumor cells in many of the cancers is composed of committed progenitor cells which are short lived and are maintained by a small population of undifferentiated long lived cancer stem cells. ${ }^{18}$ Committed progenitor cells in leukemia may acquire stem cell like behavior to generate leukemic stem cells. ${ }^{19}$ In acute myeloid leukemia progenitor cell dedifferentiate to acquire stem cell like properties unlike chronic myelogenous leukemia (CML) which is stem cell disease. ${ }^{20,21}$ In the latter it is stem cell itself which is transformed rather than progenitor cell. This is the case in chronic phase of CML, however, in blast crisis dedifferentiation takes place and granulocyte-macrophage progenitor cells acquire stem cell like properties through beta catenin mutation. ${ }^{8,18,22}$

Many of the cancers show tumor cell plasticity. ${ }^{7}$ Plasticity is the potential of a differentiated cell to dedifferentiate back into a progenitor-like or stem-like state, and then to differentiate into a new differentiated state. ${ }^{17}$

This property of cancer cells has been demonstrated in glioblastoma by the works of Ronald de Pinho's group and Friedman-Morvinski D et al. ${ }^{23,24}$ Emergence and phenotype of glioblastoma is dictated by altered regulation of genetic pathways rather than cell of origin. ${ }^{24}$ Glioblastoma can originate from varieties of cells in brain including terminally differentiated cortical astrocytes and neurons. Both astrocytes and neural stem cells can give rise to glioblastoma on genetic manipulation of certain genes. The dedifferentiation in astrocytes may be so complete that they generate pluripotent stem cells which can give rise to glia as well as neurons. ${ }^{7,24}$ This can be further clarified by the following experimental example. When an astrocyte is transduced with Lentivirus carrying gene Lenti-Hrasshp53 it is reprogrammed (dedifferentiated) to a stem cell state which gives rise to tumorspheres. Tumorspheres when transplanted into mouse brain it produces gliomas. If the same astrocyte is transduced with lentivirus carrying the four genes the 'Yamaka factors', it is reprogrammed to iPSC colonies which when transplanted into mouse gives rise to teratoma (fig. 2). ${ }^{7}$

\section{TRANSDIFFERENTIATION AND DEDIFFERENTIATION}

With above discussions it has been obvious that a non-CSC can be reprogrammed or dedifferentiated to CSCs and at the same time CSCs can differentiate to various cell types within same lineage. It is also evident that CSCs not only differentiate to cells of expected lineage but it can switch over to lineage to differentiate into another lineage of cells (transdifferentiation). It can be further clarified by the example of neuronal differentiation. Normal neural stem cell gives rise to progenitor cell and differentiates into oligodendrocyte, astrocyte, neuron and endothelial cell. ${ }^{7}$ In the process of evolution of glioblastoma, as discussed previously, neurons, astrocytes and probably oligodendrocyte can dedifferentiate or reprogrammed to CSCs within glioblastoma. These dedifferentiated CSCs can now proliferate and differentiate to more transformed neurons and glial cells. The transformed neurons and astrocytes not only dedifferentiate into CSCs, they also transdifferentiate into endothelial cells. These transdifferentiated endothelial cells can again dedifferentiate into CSCs. ${ }^{7}$ In glioblastoma the tumor cells can also transdifferentiate to pericytes of the blood vessels. ${ }^{7,25}$ 


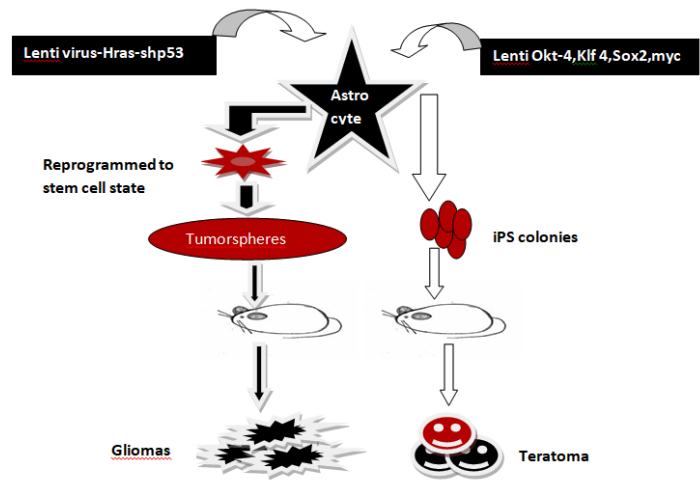

Figure 2: An acquired plasticity of astrocyte dedifferentiating to stem cells and induced pluripotent stem cell colonies (iPS) by transduction of respective oncogenes through Lentivirus. The same astrocytes depending on transduced genes can differentiate to gliomas or teratomas when transplanted into mouse brain.

\section{PATHOBIOLOGY OF DEDIFFERENTIATION}

Dedifferentiation plays a role in tumor initiation, progression, phenotypic diversity, and immortality leading to resistance to therapy. Dedifferentiation is linked with genetic and epigenetic abnormalities, as well as tumor environment. Role of hypoxia and tumor microenvironment in the immediate vicinity of solid tumors are linked with dedifferentiation.

\section{Hypoxia}

Hypoxia greatly influences cellular phenotypes by altering specific genes. It is an important contributor to intra and inter-tumor cell diversity. It is revealed by the expression of hypoxia associated genes in solid tumor. ${ }^{26}$ The oxygen tension is often lower in solid tumors than adjacent noncancerous tissue. Tumor exhibiting low oxygen tension is often aggressive than corresponding better oxygenated tumors. Hypoxic neuroblastoma cells and breast cancer cells lose their differentiated gene expression and develop stem cell phenotypes, leading to tumor dedifferentiation. Hypoxia induced tumor dedifferentiation is associated with tumor heterogeneity and aggressive behavior ${ }^{26}$ Highly aggressive, rapidly growing tumors are exposed to hypoxia or even anoxia which occurs as a consequence of inadequate blood supply. Therefore, tissue hypoxia has been regarded as a central factor for tumor aggressiveness and metastasis. Hypoxia not only accounts for tissue necrosis but has also a strong impact on tumor cell biology. ${ }^{27}$

Cancer progresses through multistep processes involving both genetic mutations and epigenetic abnormalities; however, it still remains unclear how epigenetic abnormality occurs during cancer development. Cancerpromoting inflammatory stimuli induce drastic changes in DNA methylation patterns. Thus external stimuli could be the cause of epigenetic abnormalities. ${ }^{4}$ Role of epigenetic in development of cancer has been proved in experimental

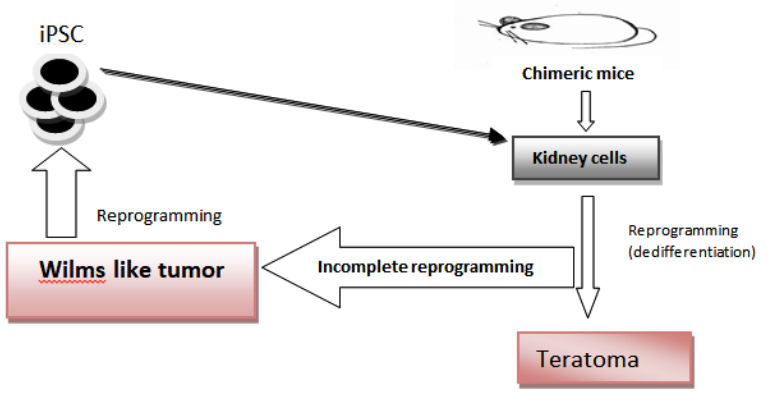

Figure 3: Experimental study showing epigenetic role in reprogramming nonneoplastic kidney cells in development of teratoma and by incomplete reprogramming into wilms' like tumor. iPSCs reprogrammed from Wilms' like tumor cells can develop into nonneoplastic kidney cells proving lack of involvement of genetic mutation

studies. Some of the cancers such as Wilms tumor may arise as a result of epigenetic disruption without detectable genetic abnormalities particular for the development of this tumor. ${ }^{4}$ In study an experimental study done in chimeric mice generated from embryonic stem cells (ESCs) shows that nonneoplastic kidney cells can be reprogrammed to develop multiple teratomas in various organs. When reprogramming process was made incomplete by withdrawing doxicycline treatment before teratoma development, the mice developed tumor resembling Wilms' tumor. In the tumor cells of the Wilms' tumor no significant genetic abnormalities detected. These tumor cells again reprogrammed to iPSCs. These tumor cell derived iPSCs again differentiated into normal kidney cells. This proves the fact that genetic changes had not occurred during this process of development of Wilms' tumor and this was as result of epigenetic alteration (fig. 3). ${ }^{4}$

Epithellial mesenchymal transition (EMT): In experimental studies first event of invasion and metastasis has been linked to dedifferentiation and dissociation of cancer cells. ${ }^{28}$ Well-differentiated carcinomas often loose epithelial differentiation and dissociate, migrate and disseminate into host stroma. This has been seen in colorectal cancers, where prognostic and metastatic activity has been reported to be associated with morphological features at invasive front of the primary tumor, which is called tumor budding or focal dedifferentiation. ${ }^{29-32}$ Barblets et al showed that tumor progression is driven by tumor environment through Wnt signal pathway. ${ }^{33}$ This type of dedifferentiation is regulated by cell to cell interactions or cell to extracellular matrix interactions at cancer invasive fronts. ${ }^{34,35}$

\section{DIAGNOSTIC IMPLICATIONS OF DEDIFFERENTIATION}

Surgical pathologists sometimes face situations when malignant tumors show features of divergent differentiation and lineage identification becomes a difficult job. Similarly there may be situation when a metastatic tumor shows 
completely unusual features without any resemblance to primary tumor. In such situations it may be difficult to take the decision to whether the secondary tumor is the same as primary with new morphology due to additional mutation or it is a different tumor unrelated to previous tumor. Such situations may be may be encountered at initial diagnosis or following therapy. Tumor biology behind such differentiation of malignant tumor needs to be understood by pathologists as well as by the clinicians for appropriate management of the patient. This phenomenon of tumor dedifferentiation is often encountered in sarcomas but in cases of carcinomas, lymphomas and melanomas, this phenomenon is not well understood and accurate diagnosis may be completely missed.

\section{Lymphoid neoplasms}

Experimental study has proven that lymphoid cells can change the lineage on genetic manipulation. The commitment of haematopoietic progenitors to the B-cell lineage and their development to mature B lymphocytes depend on the transcription factor encoded by the paired box gene 5 (Pax5). When Pax5 gene is conditionally deleted in mice mature $\mathrm{B}$ cells from peripheral lymphoid organs dedifferentiated in vivo back to early uncommitted progenitors in the bone marrow. These progenitor cells now can differentiated to $\mathrm{T}$ lymphocytes in the thymus of $\mathrm{T}$ cell deficient mice. These B-cell-derived $\mathrm{T}$ lymphocytes carried both immunoglobulin heavy- and light-chain gene rearrangements and also participated as functional $\mathrm{T}$ cells in immune reactions. Aggressive progenitor cell lymphomas developed in these mice lacking Pax5 in mature B cells which were identified by their gene expression profile. The complete loss of Pax5 in late B cells not only leads to development of lymphomas but also allows unusual plasticity of mature peripheral B cells. ${ }^{2}$

Mature B cell lymphomas such as follicular lymphoma (FL) and small lymphocytic lymphoma/chronic lymphocytic leukemia (SLL/CLL) can transform into diffuse large lymphoma of same B-cell lineage which has been well established. However, these mature B cell lymphomas can dedifferentiate into histiocytic/dendritic cell sarcoma $(\mathrm{H} /$ DS) and may follow radiation therapy. Immunophenotypic and genotypic studies is essential for diagnosis of such unconventional neoplastic evolution. A genotype identical to that of the primary B cell neoplasm in the secondary neoplasm with H/DS immunophenotype supports the lineage conversion to H/DS. A polymerase chain reaction based immunoglobulin heavy chain gene rearrangement study on FL and HS can reveal the amplicons from these two neoplasms with identical base pair size. IGH/BCL-2 fusion gene in FL and HS can be detected by dual colour dual fusion interphase fluorescence in situ analysis. ${ }^{36}$

Lymphoid neoplasm transdifferetiating to epithelial tumor is very unusual and usually not expected in routine histopathological practice. Recently a transdifferentiation of CLL into neuronedocrine carcinoma (NEC) has been reported in rectal and liver mass of the patient. Rectal mass showed composite CLL and poorly differentiated NEC while liver mass showed pure NEC. In the rectal biopsy synaptophysin and chromogranin were positive in NEC component while CLL component revealed positivity for CD20, CD5 and CD23. FISH analysis revealed Trisomy 12 in both CLL and NEC. B-cell heavy chain rearrangement study on blood sample (pure CLL) and liver metastatic NEC (pure NEC) revealed similar monoclonal peaks. Thus a clonal relation was established between CLL and NEC. It was most likely that NEC was transdifferentiated from CLL. ${ }^{37}$

\section{Melanoma}

Melanoma is one of the tumors known for morphological diversity and often mimics carcinomas, sarcomas and lymphoma. Metastatic melanoma also shows phenotypic diversity and can lose differentiation markers. Metastatic melanoma can mimic undifferentiated pleomorphic sarcoma with myxoid and giant cell components, small cell carcinoma, pleomorphic rhabdomyosarcoma, teratocarcinosarcoma with prominent rhabdomyoblasts, adenocarcinoma with feature with metaplastic bone formation. ${ }^{3}$ They may be negative for melanocytic markers such as S100, melanoma cocktail, HMB45, Melan A and SOX10; while by expressing SMA, TP53, pancytokeratin, desmin, h-caldesmon and MDM2/CDK4 further complicates the correct identification. Genotyping is a helpful surrogate marker in classifying such cases as they may show mutant BRAF, NRAS as well as BRAF/NRAS wild type. ${ }^{3}$

\section{Carcinomas}

Salivary gland carcinomas can dedifferentiate into adenocarcinoma of variable grades and undifferentiated carcinoma. The dedifferentiated components may be juxtaposed to the conventional components of respective salivary gland carcinomas. The term "High grade transformation" for adenoid cystic carcinomas (ACC) has been used by Seethala et al. This term better reflects the fact that the dedifferentiated component often maintains some features of original tumor, such as glandular differentiation. ACC also undergo transformation to adenocarcinomas which are not poorly differentiated, suggesting that the term "high grade transformation" may not be adequate. The phenomenon has also been described in acinic cell caricnoma, mucoepidermoid carcinoma, epithelial myoepithelial carcinoma, myoepithelial carcinoma and polymorphous low grade adenocarcinoma. Ki67 can help to distinguish between conventional and transformed components. Tp53 overexpression is can be uniformly seen in all the cases of transformed components, however, some 
of the cases may show mutation or deletion of Tp53. ${ }^{1}$

Dedifferentiated endometrioid adenocarcinoma is characterized by coexistence of an undifferentiated carcinoma and a low-grade endometrioid adenocarcinoma. It is often mistaken for solid component of endometrioid carcinoma and tempted to be classified under WHO scheme (amount of solid component) of grade 3 endometrioid adenocarcinoma. The dedifferentiated component is composed of solid sheets of epithelial cells and in most cases only $5-10 \%$ of the cells are positive for keratin. ${ }^{38}$

Squamous cell carcinoma can dedifferentiate into mesenchymal tumor. A case of squamous cell carcinoma with spindle cell component juxtaposed to well differentiated squamous cell carcinoma has been reported. The spindle cell component expressed vimentin and alpha SMA and lacked CK expression. ${ }^{39}$

Papillary carcinoma of thyroid dedifferentiating into anaplastic carcinoma is well established phenomenon. Anaplastic thyroid carcinoma can exist synchronously with papillary thyroid carcinoma or it may occur in patients with previous history of papillary thyroid carcinoma. ${ }^{40}$ Anaplastic transformation can also be seen at metastatic site which can confound the diagnosis. Multiple genetic abnormalities have been reported on the way to anaplastic dedifferentiation. Immunohistochemistry can be of useful in cases of metastatic tumor to identify the tumor as of thyroid origin. CK7, thyroglobulin and TTF are often positive and CK20 is negative in tumors of thyroid origin. In dedifferentiated tumors expression of thyroglobulin and TTF may be reduced or absent. ${ }^{40}$ Dedifferentiation of papillary carcinoma into squamous cell carcinoma has been reported and lineage identity can be proved by evidence of identical BRAFV600E mutation shared by both the tumors. ${ }^{41}$

\section{Meningioma}

Dedifferentiation in meningioma has to be differentiated from malignant meningioma or meningiosarcoma. Meningeosarcoma often express meningothelial markers such as EMA and vimentin. Dedifferentiation of meningioma into osteosarcoma is extremely rare event. Low grade meningothelial meningioma surrounding and well demarcated from osteosarcomatous component has been reported in a patient receiving radiotherapy for recurrent pituitary macroadenoma. The meningothelial markers were not expressed in osteosarcomatous component which were positive in the meningioma component. In addition the osteosarcomatous component expressed high proliferative index. $^{42}$

\section{Chordoma}

Chordoma is a rare bone tumor usually restricted to midline of body. Sarcomatous component in chordoma as a result of dedifferentiation is a rare event and may pose diagnostic challenge to the pathologists. Vertebral and sphenooccipital chordoma are more prone to undergo dedifferentiation than sacroccoccygeal chordoma. Dedifferentiation usually develops usually at recurrence and may or may not be associated with radiotherapy. About $6-9 \%$ of chordomas may undergo dedifferentiation. As a result of that it may be misdiagnosed as leiomyosarcoma, firbosarcoma, osteosarcoma and undifferentiated spindle cell tumor. ${ }^{43,44}$ Immunohistochemistry is useful in such cases. Chordoma shows positivity for cytokeratin (CK), epithelial membrane antigen (EMA), S100 and vimentin. MIB index is low and P53 staining is negative or low in chordomas. However, the sarcomatous component shows high MIB index, diffuse vimentin positivity, focal S100 positivity and negative staining with CK, EMA, desmin, actin, and CD34 . P53 staining is variable. ${ }^{43}$ Brachyury is a new marker which shows positivity in more than $90 \%$ of chordomas. ${ }^{43,44}$

\section{Gastrointestinal stromal tumor}

Gastrointestinal stromal tumor (GIST) is most common gastrointestinal mesenchymal tumor and often diagnosed by typical histopathological features combined with positivity for CD117 and DOG1. In addition CD34 is positive in most cases of spindle cell GIST. ${ }^{45}$ The differentiation markers may be lost when GIST transform into high grade sarcoma. ${ }^{46}$ Due to change in morphology and immunoprofile, pathologist may face diagnostic dilemma as tumor may look like non-GIST sarcoma such as angiosarcoma or rhabdomyosarcoma. ${ }^{46,47}$ The phenomenon is seen de novo or following imatinib therapy. ${ }^{46}$ In case of rhabdomyosarcomatous dedifferentiation the tumor shows loss of markers of GIST and positivity for Desmin and MyoD1 at anaplastic foci, however, the adjacent spindle cell component express markers of GIST. ${ }^{47}$ Therefore a careful evaluation with adequate sampling is warranted in cases of unusual morphology in expected GIST cases.

\section{Osteosarcoma}

Parosteal osteosarcoma is low grade variant of osteosarcoma arising from surface of bone with typical radiological and gross appearance. It can dedifferentiate into aggressive conventional high grade osteosarcoma. Parosteal osteoasrcoma is genetically as well as biologically different from conventional osteosarcoma. Dedifferentiation into telangiectatic, fibroblastic, osteoblastic and giant cell type of conventional osteosarcoma can occur. This phenomenon can occur at presentation or at recurrence. ${ }^{48}$ Correct categorization is therapeutically important as for surgery is the treatment of choice for parosteal osteosarcoma while chemotherapy is required for conventional high grade 
osteosarcoma. If diagnosis could be made preoperatively the treatment would be neoadjuvant chemotherapy, however, if diagnosis is made following resection of tumor adjuvant chemotherapy is given. Radiologically findings can be useful to arrive at correct diagnosis. Sclerotic area of parosteal osteosarcoma and lytic or cystic fluid filled areas of high grade area can be identified radiologically. A correct sampling from these radiologically different areas can offer correct diagnosis of dedifferentiated parosteal osteosarcoma. ${ }^{48,49}$

\section{Chondrosarcoma}

Dedifferentiated chondrosarcoma consists of a conventional low grade component of conventional chondrosarcoma and high grade dedifferentiated component. The latter component may show features of undifferentiated sarcoma, osteosarcoma, fibrosarcoma, rhabdomyosarcoma, leiomyosarcoma, and giant cell tumor. The dedifferentiated chondrosaroma is an aggressive neoplasm with poor prognosis.

Diagnosis is therapeutically important as chemotherapy in dedifferentiated chondrosarcoma has limited role except for control of metastatic lesions although exceptions exist. Radiological findings characteristic of conventional chondrosarcoma (matrix mineralization) and destructive lesion a feature of dedifferentiated component can be very useful in correct diagnosis. A correct pathological diagnosis is not possible if the biopsy is taken from one component ignoring the other. Radiologically mapped biopsies representing two different components can be very useful for correct preoperative diagnosis. Ki67 index can be a useful to identify the aggressive components. ${ }^{50}$

Epithelial dedifferentiation in chondrosarcoma is extremely rare event. Dedifferentiation of chondrosarcoma into squamous cell carcinoma at metastatic inguinal lymph node has been reported. Mutational analysis showed isocitrate dehydrogenase $1 \mathrm{R} 132 \mathrm{c}$ mutation in low grade chondrosarcoma component of primary tumor and squamous component of secondary in the lymph node. ${ }^{51}$

\section{Liposarcoma}

Liposarcoma (LPS) is well known for dedifferentiation. Dedifferentiation in LPS is defined as 'nonlipogenic' tumor area occupying at least one low power (10x) in proximity of well-differentiated LPS (WDLS) ${ }^{52} \mathrm{~A}$ wide spectrum of histological diverse components can be identified in the dedifferentiated LPS which pose considerable diagnostic challenge even in excised specimens. The initial smaller or incisional biopsy may be not be representative and may contain either differentiated liposarcomatous component or dedifferentiated component alone. This may lead to misclassification of the tumor depending on the component of tumor sampled. Even in the resected specimens an extensive sampling is required. Four major types of dedifferentiation can be encountered in LPS. ${ }^{52}$

Low grade dedifferentiation: In this type of dedifferentiation cellularity approaches fibromatosis, low grade fibrosarcoma and low grade myxofibrosarcoma. Nuclear atypia and mitosis are low..$^{52}$

High grade dedifferentiation: In these tumors cellularity is moderate to marked, tumor cells show considerable pleomorphism and variable mitoses. It resembles high grade fibrosarcoma or pleomorphic sarcoma. ${ }^{52}$

Heterologous dedifferentiation: The dedifferentiated component in such cases may resemble rhabdomyosarcoma or osteosarcoma. ${ }^{52}$

Homologous dedifferentiation: In these tumors lipoblastic differentiation can be identified within dedifferentiated high grade component which resembles pleomorphic liposarcoma. A pleomorphic LPS can be identified within WDLPS as a part of homologous dedifferentiation. ${ }^{52}$

In smaller biopsy tumor may be misdiagnosed according to dedifferentiated components such as rhabdomyosarcoma, follicular dendritic cell sarcoma, GIST or sometimes diagnosis may completely missed due to lack of representation. Immunohistochemistry can be of use for correct diagnosis of dedifferentiation in LPS, however, interpretation has to be made with caution. MDM2 and S100 can be useful in identification of lipoblastic cells within high grade dedifferentiated components of homologous dedifferentiation. Many of the IHC markers of LPS can be reactive in other tumors such as MDM2/CDK4 amplification can be identified in gastrointestinal stromal tumor (GIST), lipoleiomyosaroma and mesenteric fibromatosis. Similarly Beta catenin can be seen in fibromatosis. C-kit a marker of GIST can be positive in about $30 \%$ of dedifferentiated liposaroma. $^{52}$

\section{Malignant peripheral nerve sheath tumor}

Malignant peripheral nerve sheath tumor (MPNST) is derived from Schwann cells or pluripotent stem cells of neural crest and is a highly heterogeneous malignancy with divergent differentiation patterns. It has a diverse IHC patterns. A range of sarcomas and even carcinomas are included in the differential diagnosis of this tumor. Epithelioid, glandular and heterogenous components can be observed in about $15 \%$ of cases which pose considerable diagnostic challenge to diagnosing pathologist. The heterogenous components include rhabdomyoblasts, cartilage, osseous, smooth muscle, glandular, neuroendocrine and liposarcomatous components. ${ }^{53}$ It is rare to have more than two heterogenous component 
in a single MPNST, however, upto six differentiated components has been reported in a single tumor. ${ }^{53} \mathrm{~A}$ careful interpretation of morphological findings and IHC findings is required in dedifferentiated MPNST to identify masked MPNST and to differentiate this tumor from various other tumors of heterogenous cell of origin (rhabdomyoblastTriton tumor, cartilaginous, osseous, smooth muscle, glandular, neurendocrine, gangliocytic, undifferentiated sarcoma). A distinction of dedifferentiated MPNST from dedifferentiated chondrosarcoma, dedifferentiated LPS, dedifferentiated rhabdomyosarcoma and dedifferentiated osteosarcoma is not an easy task. ${ }^{53}$ Dedifferentiated components of MPNST may pose differential diagnostic challenge to differentiate from metastatic carcinoma, synovial sarcoma and mesothelioma if the location of the tumor permits. An IHC marker in isolation is not diagnostic of MPNST. Neuronal markers such as S100, CD56, and PGP 9.5 are not specific for MPNST. Nestin in combination with other markers can be of use in diagnosing MPNST. S100 expression may be lost with increasing grade of tumor and it has limited diagnostic utility. MPNST with glandular differentiation may be difficult to distinguish from synovial sarcoma and mesothelioma. S100 and cytokeratin may be expressed in both MPNST and synovial sarcoma. EMA/ CK7 for synovial sarcoma and nestin/S100 for MPNST, can be useful combination of markers. HMGA2 is expressed in MPNST but not in synovial sarcoma. Calretinin can be useful to differentiate MPNST from mesothelioma which is seldom positive in Malignant peripheral nerve sheath tumor. In addition the glandular component of MPNST may show goblet cell and neuroendocrine differentiation which is not seen in mesothelioma. ${ }^{53}$

\section{CONCLUSION}

The phenomenon of tumor dedifferentiation has been uncovered with invent of modern diagnostic molecular technology. The real nature of malignant tumor needs to be revealed for appropriate cancer therapy. All the tumors should be looked with high index of diagnostic suspicion, to know that what we are seeing under microscope is the real face of tumor or it is the mask. None of the diagnostic technology is enough if used in isolation. A combined effort by clinician, radiologist and pathologist in combination of modern technology is required to unmask the real nature of cancer.

\section{Conflict of interest: None}

\section{REFERENCES}

1. Costa AF, Altemani A, Hermsen M.Current Concepts on Dedifferentiation/High-Grade Transformation in Salivary Gland Tumors. Pathology research international 2011; Article ID 325965, 9 pages, 2011, Crossref Crossref

2. Cobaleda C, Jochum W, Busslinger M.Conversion of mature B cells into $\mathrm{T}$ cells by dedifferentiation to uncommitted progenitors. Nature. 2007;449:473-7. DOI: 10.1038/nature06159. Crossref
3. Agaimy A, Specht K, Stoehr R, et al. Metastatic Malignant Melanoma With Complete Loss of Differentiation Markers (Undifferentiated/Dedifferentiated Melanoma): Analysis of 14 Patients Emphasizing Phenotypic Plasticity and the Value of Molecular Testing as Surrogate Diagnostic Marker.Am J Surg Pathol. 2016;40:181-91. Crossref

4. Yamada I, Haga H, Yamada Y. Concise Review: Dedifferentiation meets cancer development: Proof of Concept for Epigenetic Cancer. Stem cells Transplational Medicine 2014;3:1182-87. Crossref

5. Jopling C, Boue S, Izpisua Belmonte JC. Dedifferentiation, transdifferentiation and reprogramming: three routes to regeneration. Nat Rev Mol Cell Biol. 2011;12:79-89. Crossref

6. Oku Y, Shimoji T, Takifuji K, et al. Identification of the molecular mechanisms for dedifferentiation at the invasion front of colorectal carcinoma by a gene expression analysis. Clin cancer Res 2008;14:7215-22. Crossref

7. Friedman-Morvinski D, Verma IM. Dedifferentiation and reprogramming: origins of cancer stem cells. EMBO Rep. 2014;15:244-53. Crossref

8. Daley GQ. Common Themes of Dedifferentiation in Somatic cell Reprogramming and Cancer. Cold Spring Harb Symp Quant Biol. 2008;73:171-4. Crossref

9. Pingle S. Dedifferentiation turning back the cellular clock. Scientific American. 2013. Crossref

10. Takahashi K, Yamanaka S. Induction of pluripotent stem cells from mouse embryonic and adult fibroblast cultures by defined factors. Cell. 2006;126:663-76. Crossref

11. Liu X, Huang J, Chen T, et al. Yamanaka factors critically regulate the developmental signaling network in mouse embryonic stem cells. Cell Res. 2008;18:1177-89. Crossref

12. Folmes CD, Nelson TJ, Martinez-Fernandez A, et al. Somatic oxidative bioenergetics transitions into pluripotency-dependent glycolysis to facilitate nuclear reprogramming. Cell Metab. 2011;14:264-71. Crossref

13. Panopoulos AD, Yanes O, Ruiz S, et al. The metabolome of induced pluripotent stem cells reveals metabolic changes occurring in somatic cell reprogramming. Cell Res. 2012;22:168-77. Crossref

14. Hong H, Takahashi K, Ichisaka T, et al. Suppression of induced pluripotent stem cell generation by the p53-p21 pathway. Nature. 2009;460:1132-5. Crossref

15. Utikal J, Polo JM, Stadtfeld M, et al Immortalization eliminates a roadblock during cellular reprogramming into iPS cells. Nature. 2009;460:1145-8. Crossref

16. Kumar V, Abbas AK, Aster JC. Neoplasia, In: Robbins and Cotran Pathologic Basis of Disease Vol 1. 9th edn. Reed Elsevier India: New Delhi; 2014.pp 265-340.

17. Nguyen, David H. Systems Biology of Tumor Physiology: Rethinking the Past, Defining the Future. Springer Briefs in cancer research. Springer International Publishing: Switzerland ;2016. pp21-5. Crossref

18. Jilkine A, Gutenkunst RN. Effect of Dedifferentiation on Time to Mutation Acquisition in Stem Cell-Driven Cancers. Wang E,ed. PLoS Computational Biology. 2014;10:e1003481. Crossref

19. Krivtsov AV, Twomey D, Feng Z, et al. Transformation from committed progenitor to leukemia stem cell initiated by MLL-AF9. Nature. 2006;442:818-22. Crossref

20. Goardon N, Marchi E, Atzberger A, et al. Coexistence of LMPPlike and GMP-like leukemia stem cells in acute myeloid leukemia. Cancer Cell.2011;19:138-52. Crossref

21. Zhao Z, Zuber J, Diaz-Flores E, et al.p53 loss promotes acute myeloid leukemia by enabling aberrant self-renewal. Genes Dev. 2010;24:1389-402. Crossref

22. Jamieson CH, Ailles LE, Dylla SJ, et al. Granulocyte-macrophage 
progenitors as candidate leukemic stem cells in blast-crisis CML.N Engl J Med. 2004;351:657-67. Crossref

23. Bachoo RM, Maher EA, Ligon KL, et al. Epidermal growth factor receptor and Ink4a/Arf: convergent mechanisms governing terminal differentiation and transformation along the neural stem cell to astrocyte axis. Cancer Cell.2002;1:269-77. Crossref

24. Friedmann-Morvinski D, Bushong EA, Ke E, et al. Dedifferentiation of neurons and astrocytes by oncogenes can induce gliomas in mice. Science. 2012;338:1080-4. Crossref

25. Cheng L, Huang Z, Zhou W, et al. Glioblastoma stem cells generate vascular pericytes to support vessel function and tumor growth. Cell. 2013;153:139-52. Crossref

26. Axelson H, Fredlund E, Ovenberger M, Landberg G, Påhlman S. Hypoxia-induced dedifferentiation of tumor cells-a mechanism behind heterogeneity and aggressiveness of solid tumors. Semin Cell Dev Biol. 2005;16:554-63. Crossref

27. Kunz, Ibrahim SM. Molecular responses to hypoxia in tumor cells. Molecular Cancer 2003;2:23. Crossref

28. Gabbert H. Mechanism of tumor invasion: Evidence from in vivo observations. Cancer Metastasis Rev 1985;4:293-309. Crossref

29. Ueno H, Mochizuki H, Hatsuse K, Hase K, Yamamoto T. Indicators for treatment strategies of colorectal liver metastases. Ann Surg 2000;231:59-66. Crossref

30. Ueno $\mathrm{H}$, Mochizuki $\mathrm{H}$, Hashiguchi $\mathrm{Y}$, et al. Risk factors for an adverse outcome in early invasive colorectal carcinoma. Gastroenterology 2004;127:385-94. Crossref

31. Ono M, Sakamoto M, Ino Y, et al. Cancer cell morphology at the invasive front and expression of cell adhesion-related carbohydrate in the primary lesion of patients with colorectal carcinoma with liver metastasis. Cancer1996;78:1179-86. Crossref

32. Tominaga K, Nakanishi Y, Nimura S, Yoshimura Y, Sakai Y, Shimoda T. Predictive histopathologic factors for lymph node metastasis in patients with nonpedunculated submucosal invasive colorectal cancer. Dis Colon Rectum 2005;48:92-100. Crossref

33. Brabletz T, Jung A, Reu S, et al. Variable beta-catenin expression in colorectal cancers indicates tumor progression driven by the tumor environment. Proc Natl Acad Sci USA 2001;98:10356-61. Crossref

34. Pyke C, Salo S, Ralfkiaer E, Romer J, Dano K, Tryggvason $\mathrm{K}$. Laminin-5 is a marker of invading cancer cells in some human carcinomas and is coexpressed with the receptor for urokinase plasminogen activator in budding cancer cells in colon adenocarcinoma. Cancer Res 1995;55:4132-398. Crossref

35. Masaki T, Goto A, Sugiyama M, et al. Possible contribution of CD44 variant 6 and nuclear beta-catenin expression to the formation of budding cancer cells in patient with $\mathrm{T} 1$ colorectal carcinoma. Cancer 2001;92:2539-46. Crossref

36. Maggie MS, Endi Wang. Histiocytic/Dendritic celll transformation of B cell neoplasms. Pathologic evidence of lineage conversion in differentiated Hematolymphoid malignancies. Arch Pathol and Lab Med.2013;137:865-70 Crossref

37. Castro DD. Chronic Lymphocytic Leukemia Transdifferentiates into Neuroendocrine Carcinoma: A Case Report of a New Phenomenon. N A J Med Sci. 2016;9:123-26.

38. Wu ES, Shih I-M, Díaz-Montes TP. Dedifferentiated endometrioid adenocarcinoma: An under-recognized but aggressive tumor? Gynecologic Oncology Case Reports. 2013;5:25-27. Crossref
39. Seike M, Ikeda M, Nakajima H, Kodama H. Spindle cell squamous cell carcinoma showing continuous mesenchymal dedifferentiation in a single tumor.J Dermatol. 2005;32:813-6. Crossref

40. Huang JC, Huang SC, Chou FF, Wang PW. Papillary Thyroid Carcinoma with Anaplastic Transformation: A Case Report. J Intern Med Taiwan 2009;20:167-70.

41. Acosta AM, Pins MR. Papillary thyroid carcinoma with extensive squamous dedifferentiation metastatic to the lung: BRAF mutational analysis as a useful tool to rule out tumor to tumor metastasis. Virchows Arch. 2016;468:239-42. Crossref

42. Osipov V, Ho K-C, Krouwer HG, Meyer G, Shidham VB. Postradiation dedifferentiation of meningioma into osteosarcoma. BMC Cancer. 2002;2:34. doi:10.1186/1471-2407-2-34. Crossref

43. Saito A, Hasegawa T, Shimoda T, et al. Dedifferntiated chordoma: a case report. JPN Journal of Clin Oncol 1998; 28:766-71. DOI: Crossref

44. Jambhekar NA, Rekhi B, Thorat K, Dikshit R, Agrawal M, Puri A. Revisiting chordoma with brachyury, a "new age" marker: analysis of a validation study on 51 cases. Arch Pathol Lab Med.2010;134:1181-7. Crossref

45. Swalchick W, Shamekh R, Bui MM. Is DOG1 Immunoreactivity Specific to Gastrointestinal Stromal Tumor? Cancer Control. 2015;22:498-504.

46. Antonescu CR, Zhang SRL, Nafa K, et al. Dedifferentiation in Gastrointestinal Stromal Tumor to an Anaplastic KIT Negative Phenotype - a Diagnostic Pitfall. Morphologic and Molecular Characterization of 8 Cases Occurring either de-novo or after Imatinib Therapy. Am J Surg Pathol.2013;37:385-92. Crossref

47. Jiang X, Anderson HB, Guy CD, Mosca PJ, Riedel RF, Cardona DM. Rhabdomyosarcomatous Transformation of a Gastrointestinal Stromal Tumor following Treatment with Imatinib. Case Rep Oncol Med. 2015;2015:317493. Crossref

48. Bertoni F, Bacchini P, Staals EL, Davidovitz P. Dedifferentiated parosteal osteosarcoma: the experience of the Rizzoli Institute. Cancer. 2005;103:2373-82. Crossref

49. Azura M, Vanel D, Alberghini M, Picci P, Staals E, Mercuri M. Parosteal osteosarcoma dedifferentiating into telangiectatic osteosarcoma: importance of lytic changes and fluid cavities at imaging. Skeletal Radiol.2009;38:685-90. Crossref

50. Yokota K, Sakamoto A, Matsumoto Y, Matsuda S, Harimaya K, Oda Y, Iwamoto Y. Clinical outcome for patients with dedifferentiated chondrosarcoma: a report of 9 cases at a single institute. J Orthop Surg Res.2012;7:38. Crossref

51. Zhang Y, Paz Mejia A, temple HT, Trent J, Rosenberg AE. Squamous cell carcinoma arising in dedifferentiated chondrosarcoma proved by isocytrate dehydrogenase mutation analysis. Hum Pathol. 2014;45:1541-5. Crossref

52. Rekhi B, Navale P, Jambhekar NA. Critical histopathological analysis of 25 dedifferentiated liposarcomas, including uncommon variants, reviewed at a Tertiary Cancer Referral Center. Indian journal of Pathol and Microbiol.2012;55:294-302. Crossref

53. Guo A, Liu A, Wei L, Song X. Malignant Peripheral Nerve Sheath Tumors: Differentiation Patterns and Immunohistochemical Features - A Mini-Review and Our New Findings. J Cancer. 2012;3:303-9. Crossref 